\title{
Effect of Low-Frequency Therapeutic Ultrasound on Induction of Nitric Oxide in CKD: Potential to Prevent Acute Kidney Injury
}

\author{
Michael W. Dae ${ }^{a}$ Kathleen D. Liu ${ }^{b}$ Richard J. Solomon ${ }^{c}$ Dong W. Gao ${ }^{a}$ \\ Carol A. Stillson ${ }^{\mathrm{a}}$ \\ aDepartment of Radiology and Biomedical Imaging, University of California San Francisco, San Francisco, CA, USA; \\ ${ }^{b}$ Division of Nephrology, Department of Medicine, University of California San Francisco, San Francisco, CA, USA; \\ 'Division of Nephrology and Hypertension, Department of Medicine, The Larner College of Medicine at the \\ University of Vermont, Burlington, VT, USA
}

\section{Keywords}

Nitric oxide · Contrast-induced acute kidney injury .

Low-frequency ultrasound

\begin{abstract}
Introduction: Post-contrast acute kidney injury (PC-AKI) develops in a significant proportion of patients with CKD after invasive cardiology procedures and is strongly associated with adverse outcomes. Objective: We sought to determine whether increased intrarenal nitric oxide (NO) would prevent PC-AKI. Methods: To create a large animal model of CKD, we infused 250 micron particles into the renal arteries in $56 \pm 8 \mathrm{~kg}$ pigs. We used a low-frequency therapeutic ultrasound device (LOTUS $-29 \mathrm{kHz}, 0.4 \mathrm{~W} / \mathrm{cm}^{2}$ ) to induce NO release. NO and laser Doppler probes were used to assess changes in NO content and blood flow. Glomerular filtration rate (GFR) was measured by technetium-diethylene-triamine-pentaacetic acid (Tc-99m-DTPA) radionuclide imaging. PC-AKI was induced by intravenous infusion of $7 \mathrm{~cm}^{3} / \mathrm{kg}$ diatrizoate. In patients with CKD, we measured GFR at baseline and during LOTUS using Tc-99m--DTPA radionuclide imaging. Results: In the pig model, CKD developed over 4 weeks (serum creatinine $[\mathrm{Cr}], \mathrm{mg} / \mathrm{dL}, 1.0 \pm 0.2-2.6 \pm 0.9, p<$
\end{abstract}

karger@karger.com www.karger.com/kdd

Karger ${ }^{\prime \prime} \div$

BOPEN ACCESS
(C) 2020 The Author(s)

Published by S. Karger AG, Basel

This article is licensed under the Creative Commons AttributionNonCommercial-NoDerivatives 4.0 International License (CC BYNC-ND) (http://www.karger.com/Services/OpenAccessLicense) Usage and distribution for commercial purposes as well as any distribution of modified material requires written permission.
$0.01, n=12$ ). NO and renal blood flow (RBF) increased in cortex and medulla during LOTUS. GFR increased $75 \pm 24 \%$ ( $p=$ $0.016, n=3)$. PC-AKI developed following diatrizoate i.v. infusion (Cr $2.6 \pm 0.7$ baseline to $3.4 \pm 0.6$ at $24 h, p<0.01, n=3$ ). LOTUS (starting 15 min prior to contrast and lasting for 90 min) prevented PC-AKI in the same animals 1 week later $(\mathrm{Cr}$ $2.5 \pm 0.4$ baseline to $2.6 \pm 0.7$ at $24 \mathrm{~h}, p=\mathrm{ns}, n=3$ ). In patients with CKD $(n=10)$, there was an overall $25 \%$ increase in GFR in response to LOTUS $(p<0.01)$. Conclusions: LOTUS increased intrarenal NO, RBF, and GFR and prevented PC-AKI in a large animal model of CKD, and significantly increased GFR in patients with CKD. This novel approach may provide a noninvasive nonpharmacological means to prevent PC-AKI in high-risk patients.

(c) 2020 The Author(s) Published by S. Karger AG, Basel

\section{Introduction}

Acute kidney injury (AKI) following the administration of contrast media is the 3rd leading cause of hospitalacquired renal failure and is linked to increased length of hospital stay and higher rates of mortality [1]. Data from large registries, such as the NCDR, indicate that acute 
AKI occurs in $~ 7 \%$ of all patients undergoing PCI and up to $27 \%$ of those with baseline CKD undergoing PCI [2]. Patients with preexisting renal dysfunction and microvascular insufficiency are particularly vulnerable as are patients with ST-elevation myocardial infarction [1]. Other than intravenous isotonic fluid administration and reducing the amount of contrast used, there is no guideline-recommended therapy to prevent this costly outcome [1].

A leading factor in the pathogenesis of contrast-induced AKI is the induction of endothelial dysfunction and altered renal microcirculation upon acute exposure to contrast media [3]. The endothelial dysfunction is associated with downregulation of renal cortical and medullary nitric oxide (NO) synthesis by endothelial nitric oxide synthase (eNOS) [4]. A large body of evidence supports the role of the vasodilator, $\mathrm{NO}$, as a renoprotective agent $[5,6]$. Methods to enhance NO production in the kidney prior to and following ischemic or toxic insults may provide significant therapeutic potential.

Endothelial cell NOS is activated by several stimuli, including mechanical forces such as shear stress [7]. Previous investigations have demonstrated that low-frequency ultrasound at low intensities increased NO production through upregulation of eNOS by acoustic energy-mediated shear stress [8-10]. Ultrasound in the low-frequency range has excellent tissue penetration with little attenuation or heating, making it well suited for potential clinical applications [8]. In this study, we present the results of the use of low-frequency therapeutic ultrasound (LOTUS) to generate nitric oxide, improve glomerular filtration rate (GFR) in $\mathrm{CKD}$, and to prevent AKI.

\section{Methods}

\section{Preclinical Animal Studies}

The investigation conforms with the Guide for the Care and Use of Laboratory Animals published by the National Institutes of Health (NIH Publication No. 85-23). All protocols were approved by the Institutional Committee on Animal Research. Pigs of either sex weighing 30-60 kg were preanesthetized with IM telazol and atropine, intubated, and mechanically ventilated with a mixture of isoflurane (1-4\%) and oxygen. ECG, arterial pressure, and oxygen saturation were monitored continuously. A previously characterized porcine remnant kidney model of chronic renal insufficiency was used [11]. With the pig in the supine position, bupivacaine was injected at the insertion site for femoral catheter placement, and a femoral artery was cannulated percutaneously. The left renal artery was cannulated under fluoroscopy using a $5.0 \mathrm{~F}$ tapered angled Glide catheter. Through this catheter, 150-250 $\mu \mathrm{m}$ polyvinyl alcohol particles (PVA Contour; Boston
Scientific, Boston, MA, USA) were infused until the left renal artery was completely occluded, confirmed by angiography. Next, the right upper or lower-pole renal artery was cannulated and embolized in a similar fashion. After the embolization procedure, the pig was extubated, monitored postoperatively, and treated for 3 days with antibiotics to prevent infection and started on a normal diet.

Four weeks post-embolization, the animals were anesthetized as above and used to evaluate the physiological responses to transcutaneous LOTUS. LOTUS was delivered via the Timi3 Systems Ultrasound System (Timi3 Systems, Inc., Santa Clara, CA, USA). This device delivered low-frequency $(29 \mathrm{kHz})$ ultrasound. Acoustic output was pulsed for $12-\mathrm{ms}$ duration at a pulse repetition rate of $25 \mathrm{~Hz}$, yielding peak temporal average intensity of $0.12 \mathrm{~W} / \mathrm{cm}^{2}$ and a spatial peak pulse average intensity of $0.38 \mathrm{~W} / \mathrm{cm}^{2}$.

Renal microcirculation was measured with laser Doppler flowmetry. Under isoflurane anesthesia, the remnant kidney (partially embolized right kidney) was exposed through a flank incision. A needle laser Doppler probe $(0.15 \mathrm{~mm}$ fiber separation, $780 \mathrm{~nm}$ wavelength; Perimed AB, Jarfalla, Sweden) was inserted to $\sim 15$ $\mathrm{mm}$ in depth inside the kidney mass to localize the tip in the midmedullary region. A separate single-fiber needle probe was inserted into the cortex to a depth of $5 \mathrm{~mm}$ to measure mid-cortical blood flow. Separate needle nitric oxide (NO) sensors (amiNO-IV, $700 \mu \mathrm{m}$ in diameter; Innovative Instruments, Inc., Tampa, FL, USA) were inserted 5 and $15 \mathrm{~mm}$ into the kidney mass to measure cortical and medullary NO production. After stabilization, cortical and medullary blood flow and NO concentration were measured at baseline and following application of LOTUS. At the end of the experiments, the kidney was removed and dissected to confirm the location of the tips of the needle probes.

To assess response of renal function to LOTUS, measurement of individual kidney and total GFR by radionuclide technetiumdiethylene-triamine-pentaacetic acid (Tc-99m-DTPA) imaging was done by the Gates method [12]. A 2-4 mCi dose of Tc-99mDTPA was injected at baseline during gamma camera imaging. Following 15 min of LOTUS, a 10-12 mCi dose of Tc-99m-DTPA was injected during imaging to measure change in GFR.

To assess the ability to prevent post-contrast acute kidney injury (PC-AKI), animals were studied 4 weeks post-embolization. Under isoflurane anesthesia, a venous blood sample was obtained for measurement of serum creatinine $(\mathrm{Cr})$, followed by the injection of $7 \mathrm{~cm}^{3} / \mathrm{kg}$ of diatrizoate meglumine i.v. The animals were allowed to recover, and venous blood samples were obtained to measure serum $\mathrm{Cr}$ at 24 and $48 \mathrm{~h}$ after contrast injection. One week later, the animals were returned to the laboratory, and under isoflurane anesthesia, a venous blood sample was obtained for baseline serum Cr measurement. LOTUS was started, and $7 \mathrm{~cm}^{3} / \mathrm{kg}$ of contrast was administered i.v. following $15 \mathrm{~min}$ of LOTUS, which was continued for $90 \mathrm{~min}$. The animals were allowed to recover, and venous blood samples were obtained for serum $\mathrm{Cr}$ measurement at 24 and $48 \mathrm{~h}$ post-contrast injection.

\section{Clinical Studies}

A prospective, non-randomized, single-center pilot study was done to evaluate whether LOTUS affects kidney function in $10 \mathrm{pa}$ tients with CKD. This study was determined to be a nonsignificant risk study through the University of California San Francisco's Human Research Protection Program Committee on Human Research (IRB\# 12-10471). 


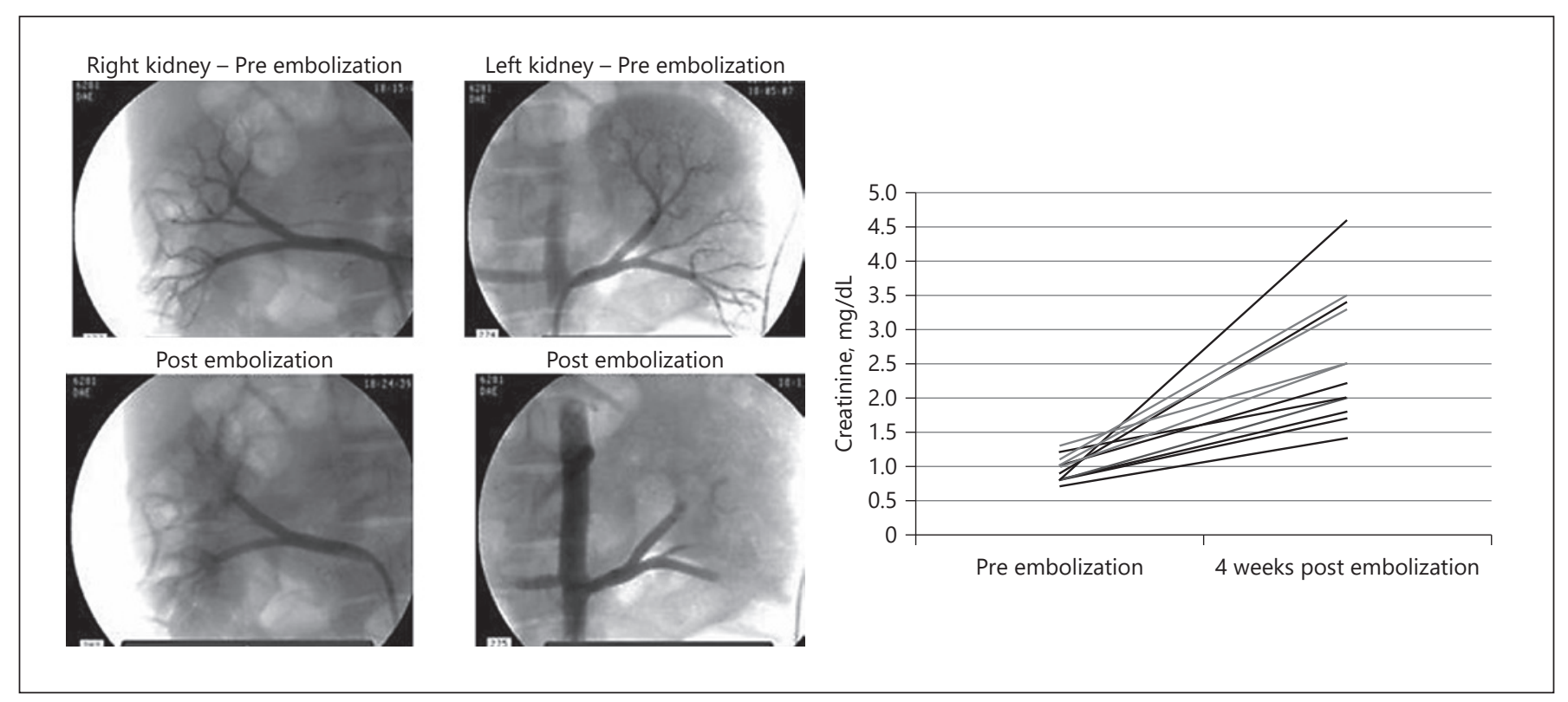

Fig. 1. (Left) Arteriograms of renal flow pre-embolization in right and left kidney (above). Post-embolization arteriograms show decreased perfusion of the upper pole of the right kidney, and total occlusion of flow to the left kidney (below). (Right) Serial changes in serum creatinine from baseline (pre-embolization) to 4 weeks postembolization.

All patients received and completed an informed consent form prior to being enrolled in the study. All participants provided written informed consent, and the study adhered to the Declaration of Helsinki. Patients $\geq 18$ years of age with Stage 3 and 4 CKD (eGFR 15-59) were enrolled. This study replicated the methods used in the preclinical study outlined above. Kidney function (GFR) was measured using Tc-99m-DTPA radionuclide imaging at baseline and after application of LOTUS. LOTUS was applied to both kidneys in the subjects for $15 \mathrm{~min}$. In addition, a 30-day telephone follow-up was conducted to ensure no latent adverse events occurred. Heart rate and blood pressure were measured to assess hemodynamic changes due to LOTUS.

\section{Statistical Analysis}

All data are expressed as mean \pm SD. Statistical analyses for comparison of changes in plasma Cr concentration and GFR before and after LOTUS were conducted by paired $t$ test. A probability value of $<0.05$ was considered statistically significant.

\section{Results}

Embolization to obliterate flow to the left kidney, and to partially obliterate flow to the right kidney, resulted in characteristics similar to a partial nephrectomy model commonly used in small animals. In $56 \pm 8 \mathrm{~kg}$ pigs, stable CKD developed over the ensuing 4 weeks, with mean serum $\mathrm{Cr}(\mathrm{mg} / \mathrm{dL})$ increasing from $1.0 \pm 0.2$ to $2.6 \pm 0.9$ $(p<0.01, n=12)$ (Fig. 1).

Nitric Oxide and Prevention of Acute Kidney Injury
During LOTUS in an invasive study, nitric oxide-sensing probes showed a significant increase in NO production in medulla and cortex. Laser Doppler probes showed an increase in microvascular blood flow in medulla and cortex in parallel with the increase in NO production (Fig. 2). Similar responses were seen in the cortex and medulla of all animals studied.

Kidney function (GFR) was measured using Tc-99mDTPA radionuclide imaging techniques at baseline and during LOTUS (Fig. 3). There was a significant increase in individual kidney GFR $(\mathrm{mL} / \mathrm{min})$ in response to LOTUS $(26 \pm 11-44 \pm 17, n=6, p<0.01)$ and total GFR (mL/ $\min )(52 \pm 13-89 \pm 14, n=3, p=0.016)$.

Intravenous infusion of $7 \mathrm{~cm}^{3} / \mathrm{kg}$ of diatrizoate increased serum $\mathrm{Cr}(\mathrm{mg} / \mathrm{dL})$ from $2.6 \pm 0.7$ at baseline to $3.4 \pm 0.6$ at $24 \mathrm{~h}(p<0.01, n=3)$, consistent with the development of PC-AKI ( $>25 \%$ increase in baseline $\mathrm{Cr}$ ). The same animals were given $7 \mathrm{~cm}^{3} / \mathrm{kg}$ of diatrizoate 1 week later, but following application of LOTUS starting $15 \mathrm{~min}$ before contrast and lasting for a total of $90 \mathrm{~min}$ (Fig. 4). LOTUS prevented the increase in serum $\mathrm{Cr}(\mathrm{Cr}$ $2.5 \pm 0.4$ at baseline to $2.6 \pm 0.7$ at $24 \mathrm{~h}, p=\mathrm{ns}, n=3$ ), consistent with the prevention of PC-AKI.

Figure 5 shows a CT scan of one of the patients, overall GFR responses to LOTUS in all patients, and systemic hemodynamic responses. There was an overall $25 \%$ in- 


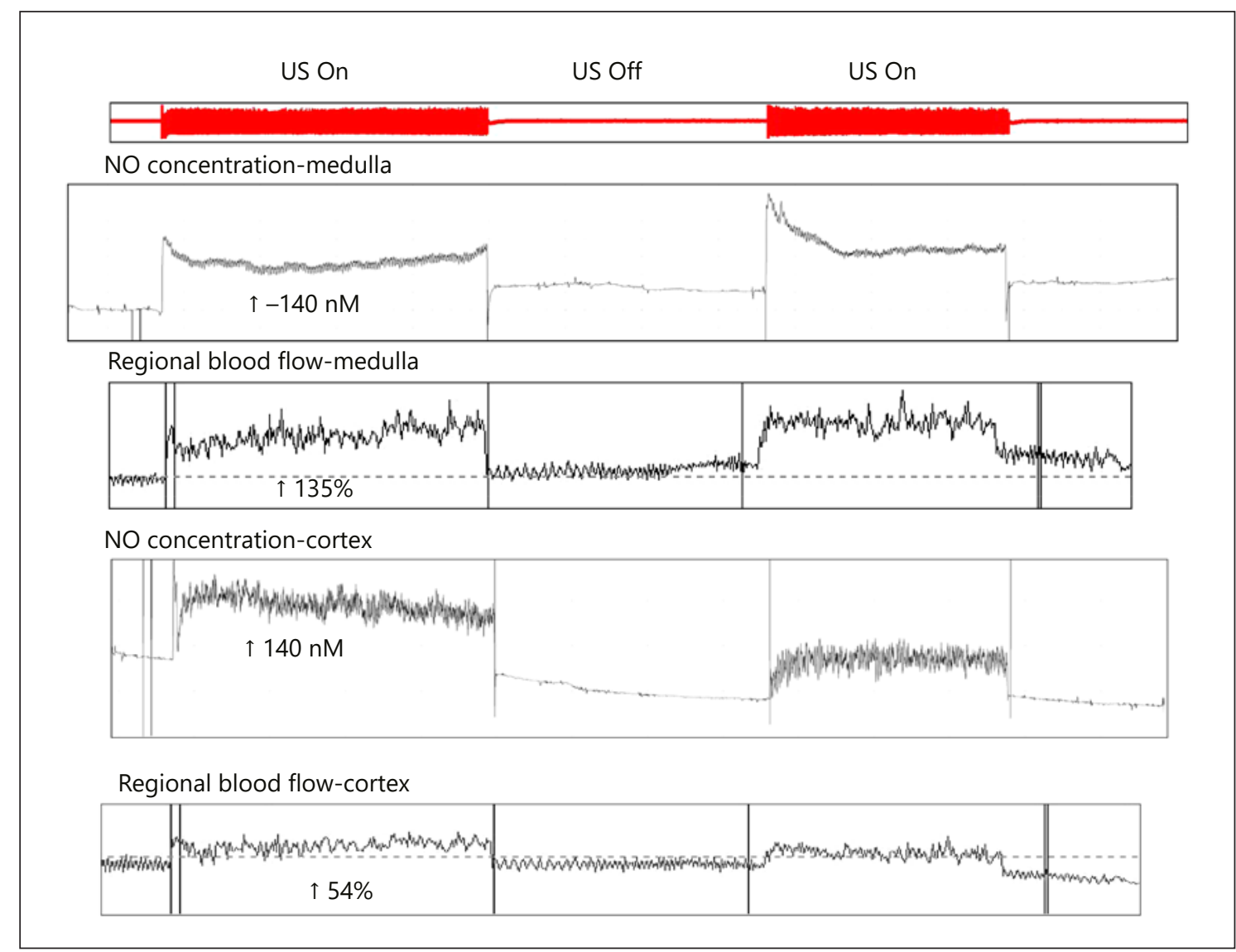

Fig. 2. With LOTUS on (thick red line, duration $15 \mathrm{~min}$ ), NO concentration in the medulla and cortex increased. Microvascular blood flow also increased in medulla and cortex during LOTUS. LOTUS, low-frequency therapeutic ultrasound device.
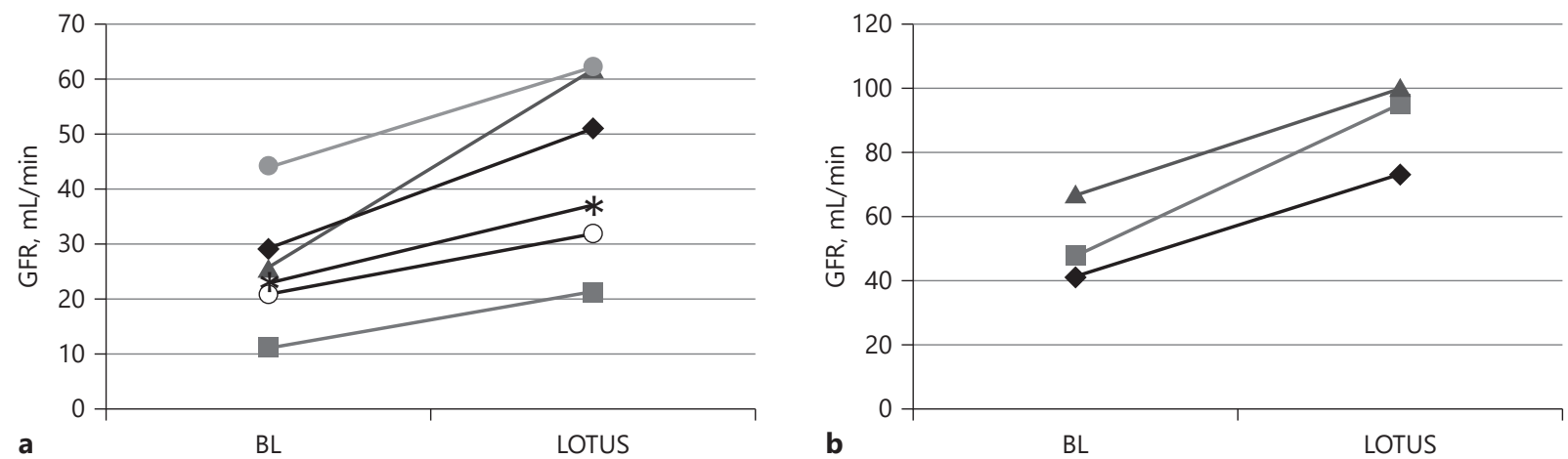

Fig. 3. a Changes in GFR for each kidney $(n=6)$ in 3 pigs at baseline $(\mathrm{BL})$ and during LOTUS. $\mathbf{b}$ Total GFR change in response to LOTUS. Total GFR increased an average of $75 \pm 25 \%,(p=0.016)$. LOTUS, low-frequency therapeutic ultrasound device; GFR, glomerular filtration rate. 
Fig. 4. Serial changes in serum Cr following intravenous diatrizoate (Pre-LOTUS in blue, Post-LOTUS 1 week later in red). The increase in serum $\mathrm{Cr}$ at $24 \mathrm{~h}$ Pre-LOTUS was blocked with the application of LOTUS. Cr, creatinine; LOTUS, low-frequency therapeutic ultrasound device.

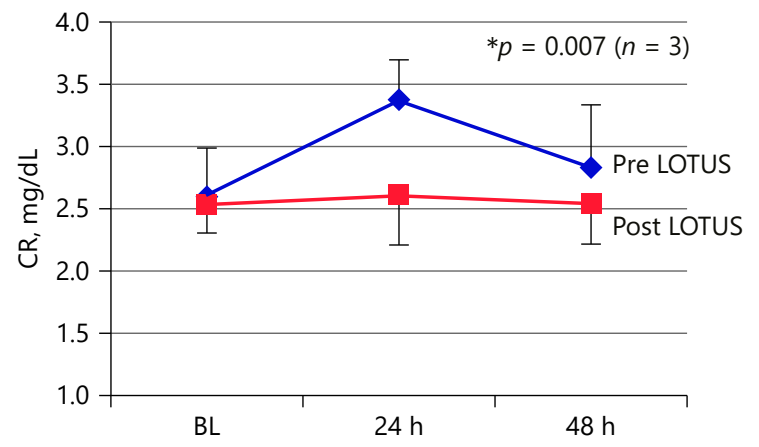

GFR
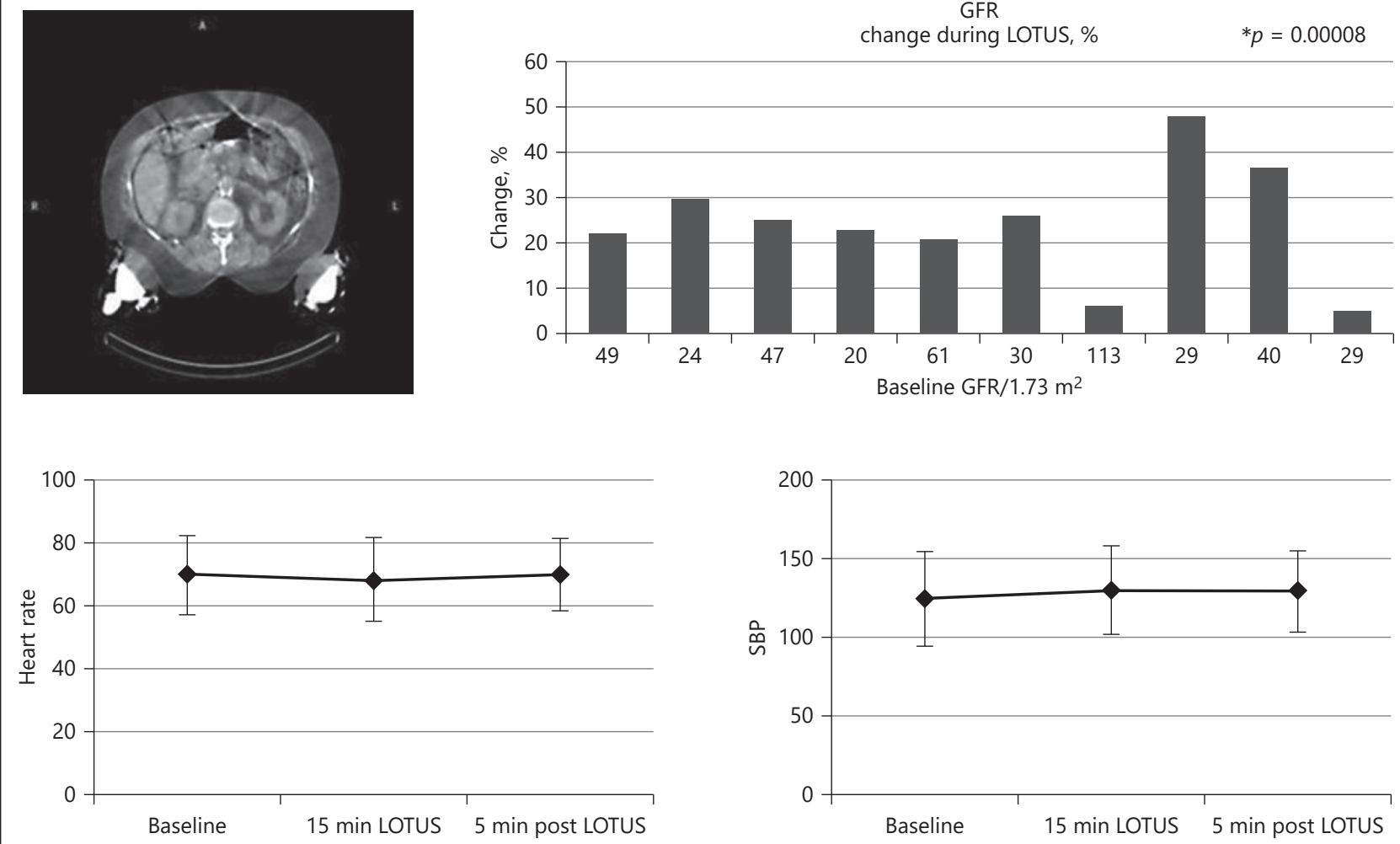

Fig. 5. CT scan showing alignment of the transducers with the kidneys. Percent change in GFR relative to baseline GFR in patients with CKD (upper right). Mean changes in heart rate and blood pressure at baseline, during, and postLOTUS are shown below. GFR, glomerular filtration rate; LOTUS, low-frequency therapeutic ultrasound device.

crease in GFR in patients with CKD during treatment with LOTUS $(p<0.01)$. There were no adverse events reported during the study. No subjects experienced a significant change in heart rate or blood pressure during or after LOTUS. All subjects completed the 30-day followup with no reported adverse events.

Nitric Oxide and Prevention of Acute Kidney Injury

\section{Discussion}

$\mathrm{AKI}$ is a common consequence of acute illness and is associated with high in-hospital morbidity and mortality [1]. A recent meta-analysis of 82 studies comprising 2,017,437 participants showed that individuals with AKI 
were at increased risk of new or progressive CKD (HR 2.67, 95\% CI: 1.99-3.58), ESRD (HR 4.81, 95\% CI: 3.047.62), and death (HR 1.80, 95\% CI: 1.61-2.02) [13]. Patients undergoing angiography procedures and those with stage 3 AKI were at the highest risk [13]. There are over 2,000,000 cardiac angiographies performed yearly in the USA alone, and the incidence of AKI continues to increase [1]. Currently, prevention is the mainstay of therapy as there is no effective therapy for AKI after it has occurred. Other than administration of intravenous isotonic fluid and limitation of contrast use, there is no effective therapy to prevent this costly outcome.

A factor in the pathogenesis of this form of AKI is the induction of endothelial dysfunction and altered renal microcirculation upon acute exposure to contrast media [3]. The endothelial dysfunction is associated with a contrast media-induced downregulation of renal cortical and medullary nitric oxide (NO) synthesis by eNOS [4]. NO functions as a vasodilator and "endogenous diuretic," which regulates medullary perfusion, salt and water handling by adjacent nephrons, and prevents ROS-mediated endothelial cell injury and blunts flow- and transport-dependent ROS formation in kidney cells $[14,15]$. The renal inner medulla, one of the most sensitive regions of the kidney to injury from contrast media, possesses the greatest amount of eNOS enzymatic activity in the kidney $[16,17]$. NO counteracts the major injury producing adverse effects of contrast media - ischemic injury and cell toxicity due to production of ROS [18]. Few therapeutic or preventative options exist for AKI. Methods to enhance NO production in the kidney prior to and following ischemic or toxic insults may provide significant therapeutic potential.

Ultrasound is cyclic sound pressure with a frequency greater than the upper limit of human hearing, which is $~ 20$ $\mathrm{kHz}$. Numerous investigations have demonstrated the use of ultrasound to accelerate fibrinolysis in vitro and in animal models [19-22]. In experiments using a rabbit femoral artery thrombolysis model, Suchova et al. [19] observed that muscle distal to a thrombus lost its cyanotic appearance during ultrasound exposure, independent of fibrinolysis and reperfusion of the femoral artery. In subsequent experiments, they showed that application of $40 \mathrm{kHz}$ ultrasound at low intensity improved perfusion and reversed acidosis in acutely ischemic skeletal muscle through a nitric oxide-dependent mechanism. They showed a 3.6-fold increase in eNOS activity concurrent with ultrasound, which was blocked with prior administration of l-NAME, an eNOS inhibitor [23]. Endothelial cell NOS is activated by several stimuli, including mechanical forces such as shear stress, the likely mechanism related to low-frequency ultrasound exposure [7].
Siegel et al. [8] showed that low-frequency, low-intensity ultrasound improved tissue perfusion and reversed acidosis in the presence of a fixed coronary occlusion in dog and pig myocardium. The effects were blocked by lNAME, again, consistent with a nitric oxide (NO)-dependent mechanism. In vitro, cultured human endothelial cells exposed to low-frequency ultrasound at low intensities increased NO production through upregulation of eNOS [9]. Sugita et al. [10] showed the direct and realtime measurement of NO generated in ischemic muscle in vivo by ultrasound alone. There was a direct relationship between the magnitude of NO produced and ultrasound intensity. The mechanism of ultrasonic NO generation is considered to be related to stimulation of the endothelium caused by acoustic energy-mediated shear stress [10]. Ultrasound in the low-frequency range has excellent tissue penetration with little attenuation or heating, making it well suited for potential clinical applications [8].

In this study, LOTUS increased the regional concentration of NO in the renal cortex and medulla in a large animal model of CKD. This was associated with increases in cortical and medullary renal blood flow. In addition, there was a significant increase in acute renal function (GFR). Further, LOTUS demonstrated the ability to prevent PC-AKI in this large animal model of CKD. This prophylactic therapy has the advantage of minimizing the time required for application before and after the administration of contrast. Unlike administration of intravenous fluid, which requires many hours of therapy before and after contrast administration, LOTUS was initiated only 15 min before contrast administration and was effective in preventing a decline in GFR when applied for only $90 \mathrm{~min}$ in total. This would translate into major cost saving and improve our therapeutic approach to patients undergoing emergency procedures, such as those with ST-elevation myocardial infarction. In patients with CKD, LOTUS increased GFR, consistent with the results found in the animal model of CKD, and supporting the idea that NO was increased in response to LOTUS. The increase in GFR with acute application of LOTUS is likely transient, returning to baseline after discontinuation. A clinical study that evaluated vasodilation effects of LOTUS in human brachial arteries demonstrated that the effect was rapid and returned to baseline about $20 \mathrm{~min}$ after discontinuation of LOTUS [24]. Shear stress-mediated NO production is mediated by an increase in eNOS protein activity that occurs within seconds due to eNOS protein phosphorylation. However, following prolonged and repeated stimulation, increases in transcription and eNOS mRNA may allow for sustained NO production [25]. Endothelialderived NO is an important mediator of angiogenesis [26] 
and has been shown to play a role in slowing interstitial fibrosis in a model of obstructed kidney [27]. Hence, repeated stimulation by LOTUS may become an effective therapeutic strategy for improving angiogenesis in patients with CKD.

\section{Conclusion}

This novel noninvasive approach may represent a nonpharmacological means to increase NO that will not only provide benefit to patients at risk for injury, but also allow patients in need of PCI, but currently denied therapy because of increased risk of kidney failure, to receive needed treatment for coronary disease. There is also an urgent need to develop therapeutic interventions to preserve renal function in patients with CKD. We speculate that this same noninvasive approach may also impact CKD progression.

\section{Statement of Ethics}

The protocol and informed consent forms were approved by the University of California San Francisco Human Research Protection Program, Committee on Human Research - IRB\# 1210471. All patients received and completed an informed consent form prior to being enrolled in the study.

\section{Conflict of Interest Statement}

M.W.D. is a co-founder, chief medical officer, director, stockholder, and patent holder for Sonogenix. R.J.S. is a paid consultant to Sonogenix. K.D.L., D.W.G., and C.A.S. report no conflicts of interest to declare.

\section{Funding Sources}

This research was supported by the University of California Discovery Grants Program, Grant No. 192935, as part of the Industry-University Cooperative Research Program in collaboration with Sonogenix, Inc., sponsor. The UC Discovery Grants Program had no role in the design of the study, nor in the collection, analysis, or interpretation of the data or in manuscript preparation.

\section{Author Contributions}

M.W.D., K.D.L., R.J.S., D.W.G., and C.A.S. made substantial contributions to the conception and design, acquisition, analysis, and interpretation of data. M.W.D., K.D.L., and R.J.S. were involved in writing and revising the manuscript. All authors gave final approval to the manuscript.

\section{References}

1 Brown JR, Rezaee ME, Nichols EL, Marshall EJ, Siew ED, Matheny ME. Incidence and inhospital mortality of acute kidney injury (AKI) and dialysis-requiring AKI (AKI-D) after cardiac catheterization in the national inpatient sample. J Am Heart Assoc. 2016;5(3): e002739.

2 Tsai TT, Patel UD, Chang TI, Kennedy KF, Masoudi FA, Matheny ME, et al. Contemporary incidence, predictors, and outcomes of acute kidney injury in patients undergoing percutaneous coronary interventions: insights from the NCDR Cath-PCI registry. JACC Cardiovasc Interv. 2014;7(1):1-9.

3 Sendeski M, Patzak A, Pallone TL, Cao C, Persson AE, Persson PB. Iodixanol, constriction of medullary descending vasa recta, and risk for contrast medium-induced nephropathy. Radiology. 2009;251(3):697-704.

4 Myers SI, Wang L, Liu F, Bartula LL. Iodinated contrast induced renal vasoconstriction is due in part to the downregulation of renal cortical and medullary nitric oxide synthesis. J Vasc Surg. 2006;44(2):383-91.

5 Ahmad A, Dempsey S, Daneva Z, Azam M, Li $\mathrm{N}, \mathrm{Li} \mathrm{PL}$, et al. Role of nitric oxide in the cardiovascular and renal systems. Int J Mol Sci. 2018;19(9):2605.
6 Phillips L, Toledo AH, Lopez-Neblina F, Anaya-Prado R, Toledo-Pereyra LH. Nitric oxide mechanism of protection in ischemia and reperfusion injury. J Invest Surg. 2009;22(1): 46-55.

7 Traub O, Berk BC. Laminar shear stress: mechanisms by which endothelial cells transduce an atheroprotective force. Arterioscler Thromb Vasc Biol. 1998;18(5):677-85.

8 Siegel RJ, Suchkova VN, Miyamoto T, Luo H, Baggs RB, Neuman Y, et al. Ultrasound energy improves myocardial perfusion in the presence of coronary occlusion. J Am Coll Cardiol. 2004;44(7):1454-8.

9 Altland OD, Dalecki D, Suchkova VN, Francis CW. Low-intensity ultrasound increases endothelial cell nitric oxide synthase activity and nitric oxide synthesis. J Thromb Haemost. 2004;2(4):637-43.

10 Sugita Y, Mizuno S, Nakayama N, Iwaki T, Murakami E, Wang Z, et al. Nitric oxide generation directly responds to ultrasound exposure. Ultrasound Med Biol. 2008;34(3):48793.

11 Misra S, Gordon JD, Fu AA, Glockner JF, Chade AR, Mandrekar J, et al. The porcine remnant kidney model of chronic renal insufficiency. J Surg Res. 2006;135(2):370-9.
12 Gates G. Split renal function testing using Tc$99 \mathrm{~m}$ DTPA. A rapid technique for determining differential glomerular filtration. Clin Nucl Med. 1983;8(9):400-7.

13 See EJ, Jayasinghe K, Glassford N, Bailey M, Johnson DW, Polkinghorne KR, et al. Longterm risk of adverse outcomes after acute kidney injury: a systematic review and metaanalysis of cohort studies using consensus definitions of exposure. Kidney Int. 2019; 95(1):160-72.

14 Zhong Z, Pallone T. Response of descending vasa recta to luminal pressure. Am J Physiol Renal Physiol. 2004;287(3):F535-42.

15 Hong NJ, Garvin JL. Nitric oxide reduces flow-induced superoxide production via cGMP-dependent protein kinase in thick ascending limbs. Am J Physiol Renal Physiol. 2009;296(5):F1061-6.

16 Sullivan JC, Pardieck JL, Hyndman KA, Pollock JS. Renal NOS activity, expression, and localization in male and female spontaneously hypertensive rats. Am J Physiol Regul Integr Comp Physiol. 2010;298(1):R61-9.

17 Wu F, Park F, Cowley AW, Mattson DL. Quantification of nitric oxide synthase activity in microdissected segments of the rat kidney. Am J Physiol. 1999;276(6):F874-81. 
18 Heyman SN, Rosen S, Khamaisi M, Idée JM, Rosenberger C. Reactive oxygen species and the pathogenesis of radiocontrast-induced nephropathy. Invest Radiol. 2010;45(4):18895.

19 Suchova V, Baggs R, Francis C. Effect of 40$\mathrm{kHz}$ ultrasound on acute thrombolytic ischemia in a rabbit femoral artery thrombosis model. Circulation. 2000;101(19):2296-301.

20 Riggs PN, Francis CW, Bartos SR, Penney DP. Ultrasound enhancement of rabbit femoral artery thrombolysis. Cardiovasc Surg. 1997; 5(2):201-7.

21 Kornowski R, Meltzer RS, Chernine A, Vered $\mathrm{Z}$, Battler A. Does external ultrasound accel- erate thrombolysis? Results from a rabbit model. Circulation. 1994;89(1):339-44.

22 Siegel RJ, Atar S, Fishbein MC, Brasch AV, Peterson TM, Nagai T, et al. Noninvasive, transthoracic, low-frequency ultrasound augments thrombolysis in a canine model of acute myocardial infarction. Circulation. 2000;101(17):2026-9.

23 Suchkova VN, Baggs RB, Sahni SK, Francis $\mathrm{CW}$. Ultrasound improves tissue perfusion in ischemic tissue through a nitric oxide dependent mechanism. Thromb Haemost. 2002; 88(5):865-70.

24 Lika K, Luo H, Hagisawa K, Akima T, Shah PK, Naqvi TZ, et al. Noninvasive low-fre- quency ultrasound energy causes vasodilation in humans. J Am Coll Cardiol. 2006;48(3): 532-7.

25 Balligand JL, Feron O, Dessy C. eNOS activation by physical forces: from short-term regulation of contraction to chronic remodeling of cardiovascular tissues. Physiol Rev. 2009; 89(2):481-534

26 Contreras D, Robles H, Romo E, Rios A, Escalante R. The role of nitric oxide in the postischemic revascularization process. Pharmac Ther. 2006;112(2):553-63.

27 Lee J, Bae E, Ma SK, Kim SW. Altered nitric oxide system in cardiovascular and renal diseases. Chonnam Med J. 2016;52(2):81-90. 